\title{
Nonequlibrium particle and energy currents in quantum chains connected to mesoscopic Fermi reservoirs
}

\author{
Shigeru Ajisaka* and Felipe Barra \\ Departamento de Física, Facultad de Ciencias Físicas y Matemáticas, Universidad de Chile, Casilla 487-3, Santiago, Chile \\ Carlos Mejía-Monasterio \\ Laboratory of Physical Properties, Technical University of Madrid, Avenida Complutense s/n, E-28040 Madrid, Spain \\ Tomaž Prosen \\ Faculty of Mathematics and Physics, University of Ljubljana, Jadranska 19, SI-1000 Ljubljana, Slovenia \\ (Received 5 April 2012; revised manuscript received 12 June 2012; published 7 September 2012; corrected 10 September 2012)
}

\begin{abstract}
We propose a model of nonequilibrium quantum transport of particles and energy in a system connected to mesoscopic Fermi reservoirs (mesoreservoir). The mesoreservoirs are in turn thermalized to prescribed temperatures and chemical potentials by a simple dissipative mechanism described by the Lindblad equation. As an example, we study transport in monoatomic and diatomic chains of noninteracting spinless fermions. We show numerically the breakdown of the Onsager reciprocity relation due to the dissipative terms of the model.
\end{abstract}

DOI: 10.1103/PhysRevB.86.125111

PACS number(s): 05.60.Gg, 03.65.Fd, 05.30.Fk, 05.70.Ln

\section{INTRODUCTION}

Nonequilibrium systems abound in nature, and still their theoretical description poses numerous challenges to theory. Nonequilibrium steady states (NESS) describe the state of a system maintained out of equilibrium by external forces, such as gradients of temperature $T$ and chemical potential $\mu$, and are characterized by the emergence of steady flows. One might expect that a finite system connected to two infinitely extended reservoirs imposing external gradients will reach a NESS after a sufficiently long time. ${ }^{1,2}$ Nonetheless, this is not always the case, and the conditions to reach a NESS are not well understood in general. Although the problem of the existence of and approach to NESS has been discussed since the early days of statistical mechanics, rigorous results are limited to a few examples. ${ }^{3-5}$ Moreover, in nonequilibrium it is customary to try to minimize unwanted disturbances of the NESS by the contacts through which the system is coupled to the external ideal and infinite reservoirs. However, this is nearly impossible at the mesoscopic scales at which the system is coupled to reservoirs through finite leads, particularly far from equilibrium, where unexpected boundary effects may arise. $^{6,7}$

In quantum mechanics the construction of NESS requires us to consider open quantum systems, rendering the problem extraordinarily more difficult. The common setup is to consider the infinite time limit of the density matrix of the finite system $S$ coupled to two infinite reservoirs which are in thermal equilibrium at different temperatures and chemical potentials, starting from an initial separable state $\rho_{L} \otimes \rho_{S} \otimes \rho_{R}$. One can then study the properties of the density operator of the total (infinite) system ${ }^{1,2}$ or the reduced density operator for the (finite) system $S,{ }^{8}$ obtained by tracing out the reservoirs' degrees of freedom. The time evolution of the density operator is naturally determined by the Von Neumann equation. However, dealing with infinite degrees of freedom is in most cases difficult. A second approach based on the master equation of the reduced density operator is more accessible, albeit at the price of several approximations such as the Born-Markov approximation (see, e.g., Ref. 9).

In this article, we propose a conceptually different approach for studying NESS that comprises mesoreservoirs with a finite number of degrees of freedom, which in turn are thermalized by Markovian macroscopic reservoirs using a simple Lindblad equation. The mesoreservoirs model the finite leads through which the system is coupled to the reservoirs. This setup yields a computationally efficient model in which the NESS of the system and of the mesoreservoirs are mathematically accessible, allowing us to study the relaxation times of the system and mesoreservoirs, the correlations among them, and their dependence on the number of degrees of freedom. Moreover, as we shall show, our approach allows us to obtain the nonequilibrium currents without the necessity of ad hoc assumptions, such as reflectionless contacts and ideally thermalized reservoirs. It is interesting to remark that finite mesoreservoirs, in principle, allow the creation of entanglement among the different degrees of freedom of the system solely through its coupling with the mesoreservoir, ${ }^{10}$ thus making our setup much richer. This paper is organized as follows. In Sec. II, we introduce the model for a chain coupled to left and right reservoirs, and we discuss some of its properties and briefly discuss the method to study relaxation to NESS and observable averages in NESS. In Sec. III we presents our main results about the particle and energy current in the NESS. We conclude in Sec. IV.

\section{THE MODEL}

We consider a one-dimensional quantum chain of spinless fermions coupled at its boundaries to mesoreservoirs comprising a finite number of spinless fermions with wave number $k(k \in\{1, \ldots, K\})$. The Hamiltonian of the system can be written as $H=H_{S}+H_{L}+H_{R}+V$, 
where

$$
\begin{aligned}
H_{S} & =-\sum_{j=1}^{n-1}\left(t_{j} c_{j}^{\dagger} c_{j+1}+\text { H.c. }\right)+\sum_{j=1}^{n} U c_{j}^{\dagger} c_{j}, \\
H_{\alpha} & =\sum_{k=1}^{K} \epsilon_{k} a_{k \alpha}^{\dagger} a_{k \alpha}, \quad \epsilon_{k} \equiv \theta_{F}\left(k-k_{0}\right), \quad \alpha=\{L, R\}, \\
V & =\sum_{k=1}^{K} v\left(a_{k L}^{\dagger} c_{1}+a_{k R}^{\dagger} c_{n}\right)+\text { H.c., }
\end{aligned}
$$

and $\left\{t_{j}\right\}$ are the nearest-neighbor hoppings, $U$ is the on-site potential, $v$ is the coupling between the system and the mesoreservoirs, $\theta_{F}$ is the inverse density of states, and $c_{j}$ and $c_{j}^{\dagger}$ are the annihilation and creation operators for the spinless fermions of the chain, while $a_{k, \alpha}$ and $a_{k, \alpha}^{\dagger}$ are those of the left and right mesoreservoirs.

The key idea of our model is to enforce the finite mesoreservoirs to equilibrium using simple Lindblad dissipators. ${ }^{11-14}$ If the couplings to the Lindblad dissipators are small, then we can interpret these terms as coming from tracing out infinitely extended (super)reservoirs (schematically depicted in Fig. 1). Thus, the density matrix of the total setup evolves according to the many-body Lindblad equation,

$$
\begin{aligned}
\frac{d}{d t} \rho & =-i[H, \rho]+\sum_{k, \alpha, m}\left(2 L_{k, \alpha, m} \rho L_{k, \alpha, m}^{\dagger}-\left\{L_{k, \alpha, m}^{\dagger} L_{k, \alpha, m}, \rho\right\}\right) \\
L_{k, \alpha, 1} & =\sqrt{\Gamma_{k, \alpha, 1}} a_{k \alpha}, \quad L_{k, \alpha, 2}=\sqrt{\Gamma_{k, \alpha, 2}} a_{k \alpha}^{\dagger}, \quad(2) \\
\Gamma_{k, \alpha, 1} & =\gamma\left[1-F_{\alpha}\left(\epsilon_{k}\right)\right], \quad \Gamma_{k, \alpha, 2}=\gamma F_{\alpha}\left(\epsilon_{k}\right), \quad \alpha=\{L, R\},
\end{aligned}
$$

where $L_{k, \alpha, 1}$ and $L_{k, \alpha, 2}$ are operators representing the coupling to the superreservoirs, $F_{\alpha}(\epsilon)=\left(e^{\beta_{\alpha}\left(\epsilon-\mu_{\alpha}\right)}+1\right)^{-1}$ are Fermi distributions, with inverse temperatures $\beta_{\alpha}$ and chemical potentials $\mu_{\alpha}$, and $[\cdot, \cdot]$ and $\{\cdot, \cdot\}$ denote the commutator and anticommutator, respectively. Generalization to spinfull fermions (e.g., electrons) is straightforward.

The parameter $\gamma$ determines the strength of the coupling to the superreservoirs. We are interested in a regime where the physical observables are $\gamma$ independent. We note that $\gamma$, which also determines the relaxation rate of the mesoreservoirs toward equilibrium, in general depends on the temperature, chemical potential, and wave number $k$ of the mesoreservoir modes. Here we consider the simplest model possible in which

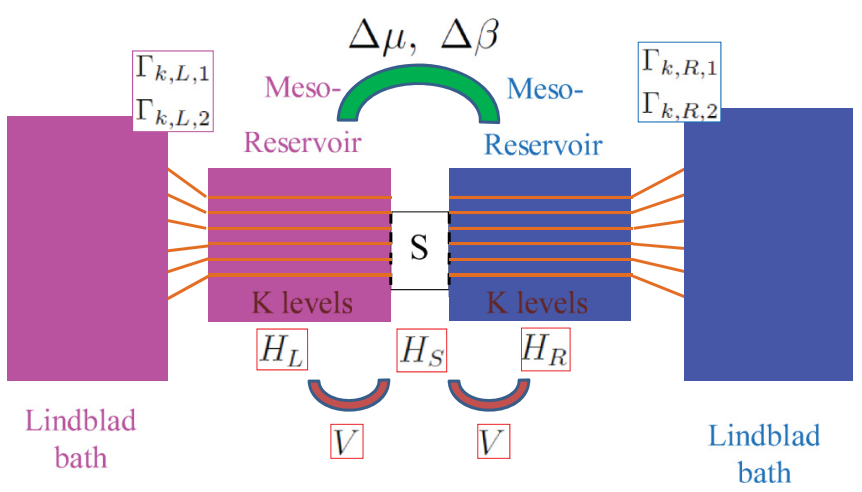

FIG. 1. (Color online) Schematic view of the two-level reservoir open-system model.
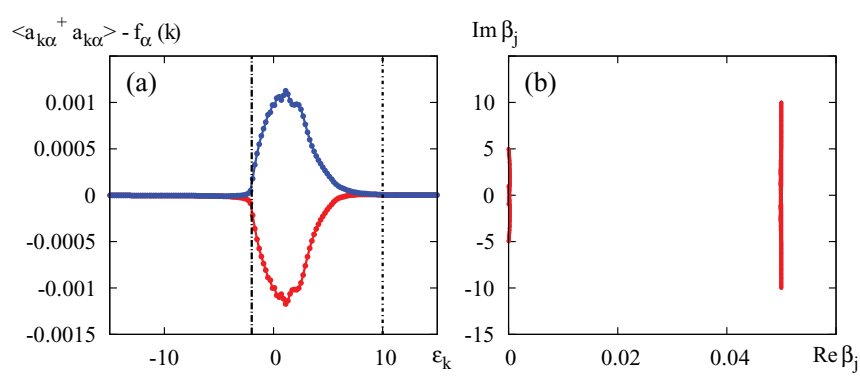

FIG. 2. (Color online) (a) The (mode) $k$ dependence of the deviation from the Fermi function $\left\langle a_{k \alpha}^{\dagger} a_{k \alpha}\right\rangle-F_{\alpha}\left(\epsilon_{k}\right)$. The red (blue) curve is for the left (right) mesoreservoir, and vertical lines indicate the energy band of the system; see main text for parameter values. (b) The rapidity spectrum for the monoatomic chain, indicating the separation of time scales.

$\gamma$ is constant. We stress that our model does not rely on the usual weak-coupling assumption needed for the physical derivation of the Lindblad master equation; ${ }^{8}$ thus $\gamma$ does not need to be a small parameter.

When the system and the mesoreservoirs are decoupled $(v=0)$, each noninteracting mode of the mesoreservoir is thermalized separately with the prescribed Fermi-Dirac occupation number. ${ }^{15}$ For small $v(v<\gamma)$, we thus expect the distribution of occupations in the mesoreservoirs to be close to Fermi-Dirac. Figure 2(a) depicts this small difference. A remarkable feature of our model is that we can monitor and control the difference of occupation distributions $\left\langle a_{k \alpha}^{\dagger} a_{k \alpha}\right\rangle-$ $F_{\alpha}\left(\epsilon_{k}\right)$ by changing the coupling parameters $v$ or $\gamma$. More important, we can show that the Lindblad equation of motion implies the following identity for averages in a steady state:

$$
\left\langle J_{k}^{\alpha}\right\rangle=-2 \gamma\left\{\left\langle a_{k \alpha}^{\dagger} a_{k \alpha}\right\rangle-F_{\alpha}\left(\epsilon_{k}\right)\right\}, \quad \alpha=\{L, R\},
$$

where $J_{k}^{\alpha}$ is the $k$ th-level contribution to the current from the $\alpha$ mesoreservoir to the system, $J_{k}^{\alpha}=i v\left(a_{k}^{\dagger} c_{\alpha}-c_{\alpha}^{\dagger} a_{k}\right)$ $\left(c_{L}=c_{1}, c_{R} \equiv c_{n}\right)$. This relation can be used to determine the Lindblad dissipator from physical observables such as currents and occupation numbers. It is interesting to remark that this relation can be interpreted as a type of Landauer formula, i.e., $\left\langle J_{\text {tot }}^{P}\right\rangle=-\sum_{k} 2 \gamma\left\{\left\langle a_{k L}^{\dagger} a_{k L}\right\rangle-F_{L}\left(\epsilon_{k}\right)\right\}$, where $J_{\text {tot }}^{P}$ is the particle current from the left reservoir to the system, and shows explicitly how the nonequilibrium situation modifies the Fermi distributions. A similar expression holds for the current from the right reservoir to the system. It can be shown that particle current is conserved. That is in NESS the average particle current inside the chain, a quantity we analyze later in detail, equal to $\left\langle J_{\text {tot }}^{P}\right\rangle$. Given our model, it is expected that this current propagates ballistically. This is directly confirmed by the numerical analysis of the particle current we present later and by the occupation density inside the system $\left\langle c_{j}^{\dagger} c_{j}\right\rangle$, which we observe to be almost a constant, i.e., independent of $j$. It follows from particle current conservation that although the distribution functions $\left\langle a_{k \alpha}^{\dagger} a_{k \alpha}\right\rangle$ are slightly modified by the presence of the system and the other reservoir, the integrated difference from the Fermi distribution summed over both mesoreservoirs satisfies $\sum_{k, \alpha}\left\{\left\langle a_{k \alpha}^{\dagger} a_{k \alpha}\right\rangle-F_{\alpha}\left(\epsilon_{k}\right)\right\}=0 .{ }^{22}$

We have studied monoatomic $\left(t_{j}=t\right)$ and diatomic $\left(t_{2 j-1}=t_{A}, t_{2 j}=t_{B}\right)$ chains. Unless specified differently, we 
have set $n=99, t=3, U=4$ for the chain. The mesoreservoirs parameters are $\epsilon_{1}=-20, \epsilon_{K}=20$, and $K=200$, fixing the spacing $\theta_{F}$ and $k_{0}$ in Eq. (1). The chain-mesoreservoir coupling is set to $v=0.03$. The superreservoirs, which enter into our model through the operators $L_{k, \alpha, 1}$ and $L_{k, \alpha, 2}$, are characterized by $\gamma=0.1$, inverse temperatures $\beta_{L}=\beta_{R}=1$, and chemical potentials that we take always with opposite values, i.e., $\mu_{L}=-\mu_{R}=\mu=3$.

\section{A. Solution method: Brief summary of third quantization}

As shown in Ref. 16, the spectrum of the evolution superoperator is given in terms of the eigenvalues $\beta_{j}$ (so-called rapidities) of the matrix $X$ :

$$
\boldsymbol{X}=-\frac{i}{2} \boldsymbol{H} \otimes \sigma_{y}+\frac{\gamma}{2}\left(\begin{array}{ccc}
\boldsymbol{E}_{K} & \mathbf{0}_{K \times n} & \mathbf{0}_{K \times K} \\
\mathbf{0}_{n \times K} & \mathbf{0}_{n \times n} & \mathbf{0}_{n \times K} \\
\mathbf{0}_{K \times K} & \mathbf{0}_{K \times n} & \boldsymbol{E}_{K}
\end{array}\right) \otimes \boldsymbol{E}_{2}
$$

where $\boldsymbol{0}_{i \times j}$ and $E_{j}$ denote an $i \times j$ zero matrix and a $j \times j$ unit matrix, $\sigma_{y}$ is the Pauli matrix, and $\boldsymbol{H}$ is a matrix which defines the quadratic form of the Hamiltonian as $H=\boldsymbol{d}^{\dagger} \boldsymbol{H} \boldsymbol{d}$ in terms of fermionic operators $\boldsymbol{d}^{T} \equiv$ $\left\{a_{1 L}, \ldots, a_{K L}, c_{1}, \ldots, c_{n}, a_{1 R}, \ldots, a_{K R}\right\}$.

Let us briefly discuss the relaxation times of our system. Figure 2(b) shows a typical rapidity spectrum for the monoatomic chain. Interestingly, there is a clear separation of the relaxation times into slow and fast normal modes. The number of slow modes $n_{s}$, with eigenvectors localized in the system part, is $n_{s} \approx 2 n$, and the number of fast modes $n_{f} \approx 4 K$, with eigenvectors localized in the mesoreservoirs. We have not observed rapidities with zero real part, which according to Refs. 15 and 16, shows that there exists a unique steady state for the range of parameters here studied.

Next, we show how the NESS averages $\langle\cdot\rangle$ of observables are computed. The quadratic observables are given in terms of the solution of the Lyapunov equation: ${ }^{16}$

$$
\begin{aligned}
\left\langle w_{j} w_{k}\right\rangle & =\delta_{j, k}-4 i Z_{j, k}, \\
w_{2 j-1} & \equiv c_{j}+c_{j}^{\dagger}, \quad w_{2 j} \equiv i\left(c_{j}-c_{j}^{\dagger}\right), \quad \boldsymbol{X}^{T} \boldsymbol{Z}+\boldsymbol{Z} \boldsymbol{X} \equiv \boldsymbol{M}_{i}, \\
\boldsymbol{M}_{i} & \equiv-\frac{i}{2} \operatorname{diag}\left\{\Gamma_{1 L}^{-}, \ldots, \Gamma_{K L}^{-}, \mathbf{0}_{1 \times n}, \Gamma_{1 R}^{-}, \ldots, \Gamma_{K R}^{-}\right\} \otimes \boldsymbol{\sigma}_{y}, \\
\Gamma_{k \alpha}^{-} & \equiv \Gamma_{k, \alpha, 2}-\Gamma_{k, \alpha, 1}=\gamma_{k \alpha}\left\{2 F_{\alpha}\left(\epsilon_{k}\right)-1\right\}, \quad \alpha=\{L, R\},
\end{aligned}
$$

whereas Wick's theorem can be used to obtain expectations of higher-order observables.

\section{NESS PROPERTIES OF PARTICLE AND ENERGY CURRENTS}

We now turn our attention to the particle and energy currents inside the chain. The particle current is defined through the conservation law of number of particles,

$$
\begin{aligned}
\frac{d c_{j}^{\dagger} c_{j}}{d t} & =J_{j-1}^{P}-J_{j}^{P}(2 \leqslant j \leqslant n-1), \\
J_{j}^{P} & \equiv i t_{j}\left(c_{j}^{\dagger} c_{j+1}-c_{j+1}^{\dagger} c_{j}\right)(1 \leqslant j \leqslant n-1),
\end{aligned}
$$

and the energy current is defined through the conservation law of local energy,

$$
\begin{aligned}
\frac{d H_{j}}{d t} & =J_{j-1}^{E}-J_{j}^{E}(2 \leqslant j \leqslant n-1), \\
J_{j-1}^{E} & =i\left[H_{j-1}, H_{j}\right](2 \leqslant j \leqslant n), \\
H_{j} & \equiv t_{j} c_{j}^{\dagger} c_{j+1}+t_{j} c_{j+1}^{\dagger} c_{j}+U c_{j}^{\dagger} c_{j}(1 \leqslant j \leqslant n),
\end{aligned}
$$

where, by definition, $c_{n+1} \equiv 0$. Both currents converge to a constant by increasing the system size $n$ for the monoatomic chains. For the diatomic chains, the currents converge to different values, depending on the parity of the length of the chain $n$. Therefore, ballistic transport is indeed achieved.

Figure 3(a) shows the $K$ dependence of the particle current for the monoatomic chains with $\gamma$ ranging from 0.0001 to 10. The particle current increases linearly with the number of mesoreservoir modes $K$, with notable fluctuations in $K$ observed for some range of $\gamma$ (which will be discussed later). We see that the particle current is roughly $\gamma$ independent for $\gamma \in[0.001,1]$ and is somewhat smaller for very small or very large $\gamma$. The dependence on $\gamma$ of the particle current, shown in Fig. 3(c), exhibits a plateau starting at $\gamma \approx v$, and we take $\gamma$ in the plateau region $[v,\|H\|]$.

Figure 3(d) shows the dependence of the particle current on the bias of the chemical potential $(\mu)$. We find, as expected, initially linear increase in $\mu$, which slows down for larger $\mu$.
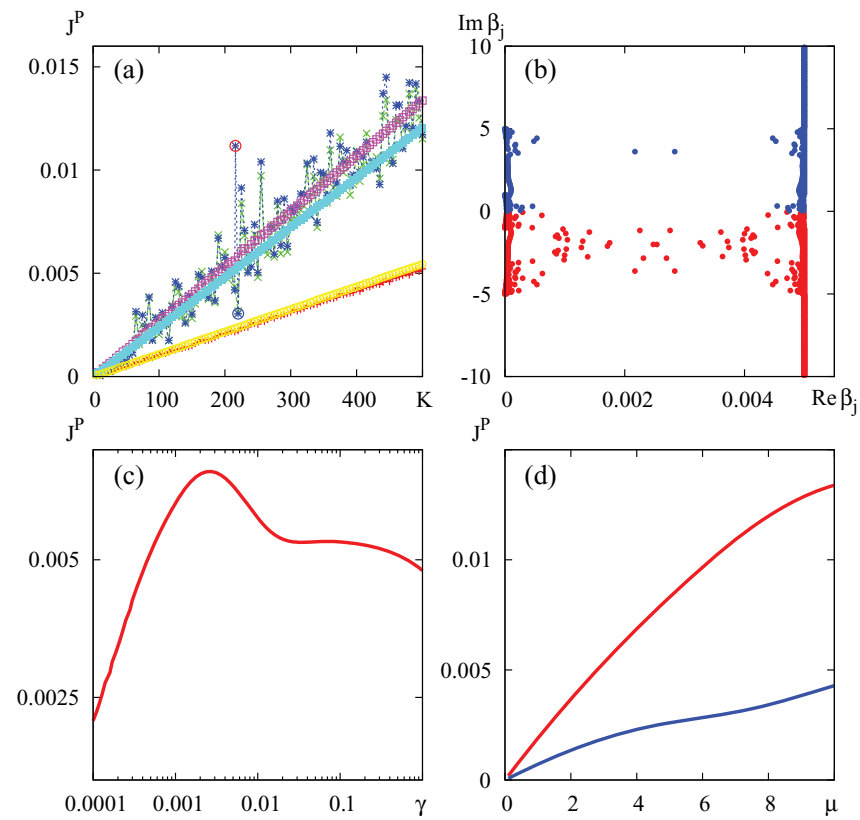

FIG. 3. (Color online) (a) The $K$ dependence of the particle current for the monoatomic chains (red: $\gamma=0.0001$, green: $\gamma=$ 0.001 , blue: $\gamma=0.01$, pink: $\gamma=0.1$, cyan: $\gamma=1$, yellow: $\gamma=10$ ) and (b) the rapidities for $\gamma=0.01$. Using the symmetry with respect to real axis, we plot rapidities in positive (negative) imaginary plane for $K=216(K=220)$. Those two parameters are indicated in (a) as red $(K=216)$ and blue $(K=220)$ circles. (c) The $\gamma$ dependence of the particle current for the monoatomic chains. (d) The $\mu=\mu_{L}=-\mu_{R}$ dependence of the particle current. The red line is for the monoatomic chains $(t=3)$, and the blue line is for the diatomic chains $\left(t_{A}=3, t_{B}=6\right)$. The values of the fixed parameters are given in the text. 
The fluctuations in the current as a function of $K$ occur because for some values of $K$ the coupling between the mesoreservoirs and system is stronger. Intuitively, this happens when an eigenenergy of the mesoreservoir coincides with one of the system, resulting in some eigenvector of the matrix $\boldsymbol{X}$ being supported both in the system and in the mesoreservoirs, breaking the separation into slow and fast modes. When the density of states of the mesoreservoir becomes dense enough such that it is smooth (average level spacing smaller than the width given by the coupling $\gamma$ to the superreservoirs), the current becomes a smooth function of $K$ or, equivalently, of the density of states. These ideas are confirmed by the numerical results in Figs. 3(a) and 3(b). We note, however, that the current fluctuations are strong only when the mesoreservoir density of states is not smooth.

Figure 4(a) shows the dependence of the particle current driven by either thermal or chemical gradients on the hopping strength $t_{B}$ for diatomic chains. Where the particle current is negative, the energy band of the chain is located entirely below the Fermi energy of the cooler reservoir, yielding a particle current flow from the cold to the hot reservoir. Such crossed transport (and its counterpart of the energy current
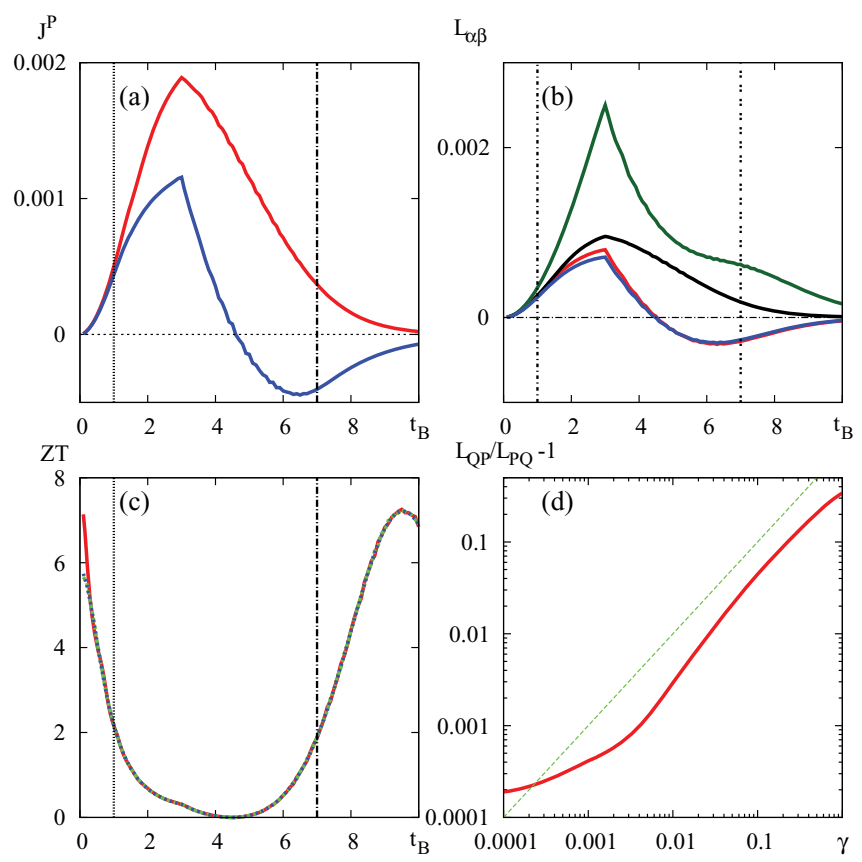

FIG. 4. (Color online) (a) The $t_{B}$ dependence of the particle current for the chains. The red line is for $\beta_{L}=\beta_{R}=1$ and $\mu=$ $\mu_{L}=-\mu_{R}=1$, and the blue line is for $\beta_{L}=0.5, \beta_{R}=4$, and $\mu=0$. (b) The $t_{B}$ dependence of the Onsager coefficients (color code: $L_{Q P}$, red; $L_{P P}$, black; $L_{Q Q}$, green; $L_{P Q}$, blue), and (c) dependence of $Z T$ [comparing $K=200$ (green) to $K=100$ (red) and $K=500$ (blue)] for the diatomic chains $\left(t_{A}=3\right)$. Chains have exponentially small current outside $t_{b} \in[1,7]$ indicated in the figure. (d) The $\gamma$ dependence of asymmetry $L_{Q P} / L_{P Q}-1$ for the diatomic chain with $t_{A}=3, t_{B}=6$ (dashed line indicates linear growth). Note that asymmetry grows linearly with $\gamma$ in the regime $\gamma>v$. In (b), (c), and (d) we have set $\mu_{L}=-\mu_{R}=0.001, \beta_{L}=\beta_{R}=1$ to compute $L_{Q P}$ and $L_{P P}$ and $\mu_{L}=\mu_{R}=0, \beta_{L}=0.999, \beta_{R}=1.001$ to compute $L_{Q Q}$ and $L_{P Q}$. driven by the chemical gradient) can be exploited to pump heat or particles. ${ }^{17}$

For sufficiently small thermal and chemical gradients, the particle and heat current defined as $J_{Q} \equiv J_{E}-\bar{\mu} J_{P}[\bar{\mu}=$ $\left.\left(\mu_{L}+\mu_{R}\right) / 2\right]$ depend linearly on the external gradients as ${ }^{18}$

$$
\begin{aligned}
& J_{Q}=L_{Q Q} \Delta \beta-\beta L_{Q P} \Delta \mu, \\
& J_{P}=L_{P Q} \Delta \beta-\beta L_{P P} \Delta \mu,
\end{aligned}
$$

where $\Delta \beta \equiv \beta_{R}-\beta_{L}$ and $\Delta \mu \equiv \mu_{R}-\mu_{L}$. In our calculation we take $\mu_{L}=-\mu_{R}=0.001, \beta_{L}=\beta_{R}=1$ to obtain $L_{Q P}$ and $L_{P P}$ and $\mu_{L}=\mu_{R}=0, \beta_{L}=0.999, \beta_{R}=1.001$ to obtain $L_{Q Q}$ and $L_{P Q}$, which are checked to be in the linear response regime. The second law of thermodynamics imposes definite positiveness of the matrix of Onsager coefficients $\boldsymbol{L}$, which implies $L_{Q Q} \geqslant 0$ and $L_{P P} \geqslant 0$, and if the dynamics is time reversible, the Onsager's reciprocity relation $L_{P Q}=L_{Q P}$ holds. In Fig. 4 we consider diatomic chains with $t_{A}=3$ and show the dependence of various properties of $\boldsymbol{L}$ on the other hopping parameter, $t_{B}$. Figure 4(b) shows the $t_{B}$ dependence of all Onsager coefficients, whereas Fig. 4(c) shows the thermoelectric figure of merit $Z T \equiv L_{P Q} L_{Q P} / \operatorname{det} \boldsymbol{L} .{ }^{19}$ One sees that while nondiagonal elements $L_{Q P}$ or $L_{P Q}$ can be sometimes negative, $L_{Q Q}$ and $L_{P P}$ are always positive. Moreover, $Z T$ reaches large values only for disproportionate hopping rates $t_{A}$ and $t_{B}$.

On the other hand, systems interacting with environments inevitably include irreversible processes, which break down time-reversibility invariance and thus the validity of the Onsager reciprocity relation. ${ }^{20}$ Figure 4(d) shows the $\gamma$ dependence of $L_{P Q} / L_{Q P}$, and we see that the relation is roughly linearly broken by increasing $\gamma$, and thus we conclude that the Onsager reciprocity relation is satisfied only if there is a time-reversible dynamics for the total system, including the superreservoirs, which is the case in which the Onsager reciprocity relation is rigorously proved. ${ }^{21} \mathrm{We}$ recall that $\gamma \ll 1$ is one of the necessary conditions for deriving the Lindblad equation by taking a partial trace of the unitary time evolution; thus $\left|L_{P Q} / L_{Q P}-1\right|$ can be understood as an error indicator due to the weak-coupling assumption. We remark that the transport coefficients for the diatomic chains [shown in Figs. 3(d) and 4] are nonsmooth functions of $t_{B}$ at the monoatomic point $t_{B}=t_{A}$.

\section{CONCLUSIONS}

We have introduced a model of an open quantum system with finite mesoreservoirs and studied nonequilibrium steady states of quantum chains. The NESS is robust with respect to the strength of the Lindblad dissipators. Moreover, we have found that the occupation number distributions of the mesoreservoirs are close to the Fermi distribution, the difference being determined by the particle current. The decay times of the normal modes show a clear separation into slow modes (with support in the system) and fast modes (with support in the mesoreservoirs). In a certain regime we observed strongly fluctuating currents (as a function of any generic parameter), which is attributed to the existence of well-separated intermediate (resonant) decay modes. We found that nondiagonal elements of the Onsager matrix can be negative but confirmed the positivity of the full Onsager 
matrix. The Onsager reciprocal relation was shown to be correct only for weak coupling $\gamma \ll 1$, and the symmetry is broken linearly as a function of $\gamma$.

\section{ACKNOWLEDGMENTS}

The authors thank J. von Delft, D. Kosov, Y. Ohta, K. Saito, and M. Žnidarič for discussions on related subjects. S.A. thanks R. Soto for the settings of computational environments and Fondecyt (Grant No. 3120254) for support. T.P. acknowledges support from Grants No. P1-0044 and No. J1-2208 from the Slovenian Research Agency. T.P. and C.M.M. acknowledge partial support from Finlombarda project "THERMOPOWER" and from the European Science Foundation through the Research Network "Exploring the Physics of Small Devices." F.B. and T.P. acknowledge international collaboration project Fondecyt 1110144. Finally, F.B. and S.A. thank project Anillo ACT 127. *g00k0056@suou.waseda.jp

${ }^{1}$ D. Ruelle, J. Stat. Phys. 98, 57 (2000).

${ }^{2}$ D. Ruelle, Commun. Math. Phys. 224, 3 (2001).

${ }^{3}$ Z. Rieder, J. L. Lebowitz, and E. Lieb, J. Math. Phys. 8, 1073 (1967).

${ }^{4}$ C. Kipnis, C. Marchioro, and E. Presutti, J. Stat. Phys. 27, 65 (1982).

${ }^{5}$ G. M. Schütz, in Phase Transitions and Critical Phenomena, edited by C. Domb and J. L. Lebowitz (Academic Press, London, 2001).

${ }^{6}$ D. Zubarev, Nonequilibrium Statistical Thermodynamics (Springer, Berlin, 1995).

${ }^{7}$ S. Sasa and H. Tasaki, J. Stat. Phys. 125, 125 (2006).

${ }^{8}$ H.-P. Breuer and F. Petruccione, Theory of Open Quantum Systems (Oxford University Press, Oxford, 2002).

${ }^{9}$ H. Wichterich, M. J. Henrich, H.-P. Breuer, J. Gemmer, and M. Michel, Phys. Rev. E 76, 031115 (2007).

${ }^{10}$ D. Braun, Phys. Rev. Lett. 89, 277901 (2002).

${ }^{11}$ V. Gorini, A. Kossakowski, and E. C. G. Sudarshan, J. Math. Phys. 17, 821 (1976).
${ }^{12}$ G. Lindblad, Commun. Math. Phys. 48, 119 (1976).

${ }^{13}$ A. A. Dzhioev and D. S. Kosov, J. Chem. Phys. 135, 174111 (2011).

${ }^{14}$ A. A. Dzhioev and D. S. Kosov, J. Chem. Phys. 134, 044121 (2011).

${ }^{15}$ T. Prosen, New J. Phys. 10, 043026 (2008).

${ }^{16}$ T. Prosen, J. Stat. Mech. (2010) P07020.

${ }^{17}$ G. Casati and C. Mejía-Monasterio, J. Korean Phys. Soc. 50, 158 (2007).

${ }^{18} \mathrm{~S}$. R. de Groot and P. Mazur, Non-equilibrium Thermodynamics (Dover, New York, 1984).

${ }^{19}$ G. Mahan, B. Sales, and J. Sharp, Phys. Today 50(3), 42 (1997).

${ }^{20}$ R. Alicki, Rep. Math. Phys. 10, 249 (1976).

${ }^{21}$ P. A. Jacquet, J. Stat. Phys. 134, 709 (2009).

${ }^{22}$ We find that a slightly more general relation is satisfied in the NESS of the system with arbitrary $K$-level mesoreservoirs; namely, having two possibly different rates of coupling to Lindblad superreservoirs, $\gamma_{L}$ and $\gamma_{R}$, we have $\sum_{k, \alpha} \gamma_{\alpha}\left\{\left\langle a_{k \alpha}^{\dagger} a_{k \alpha}\right\rangle-F_{\alpha}\left(\epsilon_{k}\right)\right\}=0$. 